Symposium on Cytology

By William L. Doyle, Hans Ris, Franz Schrader and C. Leonard Huskins. Pp. viii +69 . (East Lansing, Mich. : Michigan State College Press, 1951.) 2 dollars.

THE four papers in this symposium show four methods of discussing cytology. W. L. Doyle, with a good introduction on cell enzymes, presents his own study of phosphatases in the cells of the appendix and kidney of the rabbit. Hans Ris reviews recent work on chromosome and nuclear chemistry. He points out that the contents of samples of nuclei have been shown to be constant in respect of deoxyribose nucleic acid, and he concludes, perhaps rashly, that individual chromosomes do not vary in this respect in the mitotic cycle. Franz Schrader discusses, concisely, some differences of detail between the tactoid and protein chain hypotheses of spindle structure. He works on the assumption that they are mutually exclusive. C. L. Huskins winds up the proceedings with a pastoral discourse on the relations of "Science, Cytology and Society" combined with some observations of his own on meiosis (not mitosis) in the roots of onions.

C. D. Darlington

\section{The Classical Theory of Fields}

Translated from the Russian by Morton Hamermesh. (Addison-Wesley Physics Series.) By L. Landau and E. Lifshitz. Pp. ix + 354. (Cambridge, Mass. : Addison-Wesley Press, Inc., 1950.) 7.50 dollars.

THIS is an admirable systematic account of the 1 theory of electromagnetic and gravitational fields, which is part of a general course in classical physics. The English-speaking reader will perhaps be surprised at the omission of all references to the theory of dielectrics and of magnetic materials, but these subjects are probably discussed in another book of this series. On the other hand, he will be pleased at the prominence which is given to the special theory of relativity, which is indeed the basis of the presentation.

This treatise gives a general account of relativistic mechanics, including, of course, the motion of point charges in electromagnetic fields. Maxwell's equations are studied in detail, and are applied to steady fields, electromagnetic waves, problems of physical optics, the field of moving charges, and the general theory of the radiation of electromagnetic waves. The last two paragraphs introduce the reader to Einstein's general theory of relativity, and discuss the motion of a particle in a gravitational field, and the formation of Einstein's gravitational field equations.

The book is beautifully printed and produced, and has a good index.

\section{An Introduction to Applied Mathematics}

By Prof. J. C. Jaeger. Pp. xiii +446 . (Oxford : Clarendon Press; London: Oxford University Press, 1951.) 35s. net.

A LL those who are called upon to teach matheA matics to students of physics and engineering will feel themselves under a great obligation to Prof. J. C. Jaeger for this most useful and timely volume. The author gives an admirable survey of many, if not all, of those topics in applied mathematics which are needed by the physicist and engineer. The work covers, of course, the subject of ordinary and partial differential equations, with special reference to boundary-value problems and numerical methods. There is a most useful chapter on electrical circuit theory together with an introduction to the subject of Fourier series and integrals. Vectors, particle dynamics, rigid dynamics and Lagrange's equations each receive one chapter. There are numerous useful examples, and the whole volume is clearly written by a master of mathematical exposition.

There is perhaps just one criticism which might be levelled, and that is that no proof whatever is given of the convergence of Fourier series or integrals to the generating function. It would be most valuable to students if an elementary proof were given, even though it applied to a very restricted class of functions.

\section{Linear Polymers}

By Elizabeth M. Frith and R. F. Tuckett. Pp. xi+ 355. (London, New York and Toronto : Longmans, Green and Co., Ltd., 1951.) 18s. net.

7 HIS treatment of a rapidly growing subject is well thought out, and the authors are to be congratulated on a fine piece of work. Every section bears the unmistakable stamp of careful preparation; the preliminary marshalling of material, its digestion and thoughtful re-presentation are everywhere evident. The book is, for this reason, personal and holds the attention in spite of the high concentration of facts and theories which are to be found in it. There is, indeed, a great deal of information, critically presented, in this book. It can be warmly recommended to all research workers interested in the fundamental physical chemistry of high-polymeric systems and is especially suitable for those just entering the field.

\section{The Topology of Fibre Bundles}

By Norman Steenrod. (Princeton Mathematical Series, No. 14.) Pp. viii + 224. (Princeton, N.J.: Princeton University Press; London: Oxford University Press, 1951.) 32s. 6d. net.

7 HIS book is the first to give an account of the theory of fibre bundles, a subject which was initiated during the years 1935-40. The work of Whitney, Hopf and Stiefel indicated the importance of the subject for applications of topology to differential geometry, in particular to the study of properties 'in the large' of differentiable manifolds. The subject includes also a topological study of the coset decompositions of Lie groups by subgroups, and the theory of covering spaces used in analysis.

The author has eliminated a certain amount of confusion which had existed owing to different definitions of 'fibre bundle' being in use, as it was not clear previously that any one definition would suffice for all requirements.

The book is divided into three parts: (1) the general theory of bundles; (2) the homotopy theory of bundles; (3) the cohomology theory of bundles.

Part 1 closes with sections on covering spaces and the fundamental group. Part 2 opens with a survey of the homotopy groups of Hurewicz, since no treatment of these had previously appeared in book form. Part 3 includes a survey of cohomology theory, since the standard treatments do not contain the general. ized form required in the text.

The book is 'difficult' in the sense that considerable previous knowledge of algebraic and general topology is assumed on the part of the reader. It is, however, ably written, and is well worth a close study by algebraic topologists. The author has himself made a substantial contribution to the subject.

$$
\text { R. G. CookE }
$$

